This is the author's final, peer-reviewed manuscript as accepted for publication. The publisher-formatted version may be available through the publisher's web site or your institution's library.

\title{
Periodic and uniform nanogratings formed on cemented carbide by femtosecond laser scanning
}

Yunsong Lian, Jianxin Deng, Youqiang Xing, Shuting Lei, Xiaoming Yu

\section{How to cite this manuscript}

If you make reference to this version of the manuscript, use the following information:

Lian, Y., Deng, J., Xing, Y., Lei, S., \& Yu, X. (2013). Periodic and uniform nanogratings formed on cemented carbide by femtosecond laser scanning. Retrieved from http://krex.ksu.edu

\section{Published Version Information}

Citation: Lian, Y., Deng, J., Xing, Y., Lei, S., \& Yu, X. (2013). Periodic and uniform nanogratings formed on cemented carbide by femtosecond laser scanning. Applied Surface Science, 282, 518-524.

Copyright: @ 2013 Elsevier B.V.

Digital Object Identifier (DOI): doi:10.1016/j.apsusc.2013.06.004

Publisher's Link: http://www.sciencedirect.com/science/article/pii/S0169433213011033

This item was retrieved from the K-State Research Exchange (K-REx), the institutional repository of Kansas State University. K-REx is available at http://krex.ksu.edu 


\title{
Periodic and uniform nanogratings formed on cemented carbide by femtosecond laser scanning
}

\author{
Yunsong Lian ${ }^{\mathrm{a}}$, Jianxin Deng ${ }^{\mathrm{a}}$, Youqiang Xing ${ }^{\mathrm{a}}$, Shuting Lei ${ }^{\mathrm{b}}$, Xiaoming $\mathrm{Yu}^{\mathrm{b}}$ \\ ${ }^{a}$ School of Mechanical Engineering, Shandong University, Jinan, 250061, PR China \\ ${ }^{b}$ Department of Industrial and Manufacturing Systems Engineering, Kansas State University, \\ Manhattan, KS 66506, USA
}

Keywords: Femtosecond laser; Uniform nanogratings; Nanograting period; Cemented carbide.

Abstract. Periodic and uniform nanogratings are fabricated by femtosecond laser scanning on cemented carbide. Specifically, three experiments are designed to study the influence of single pulse energy, scanning speed, and scanning spacing on the period and the uniformity of the formed nanogratings. The results show that the sample with single pulse energy of $2 \mu \mathrm{J}$, scanning speed of $1000 \mu \mathrm{m} / \mathrm{s}$, and scanning spacing of $5 \mu \mathrm{m}$ shows the best quality of nanogratings among all the tested samples at different processing parameters. The uniformity of the nanogratings is largely determined by single pulse energy, scanning speed, and scanning spacing. Single pulse energy and scanning speed significantly affect the period of the nanogratings, whereas the period of the nanogratings maintains a fixed value under different scanning spacings. The period of the nanogratings increases gradually with the decrease of the single pulse energy and the increase of the scanning speed, respectively.

\section{Introduction}

Recent studies show that laser induced periodic surface structures (LIPSSs) on various materials (including metals [1,2], semiconductors [3,4], and insulators [5,6]) have been investigated extensively using femtosecond laser. According to the periods of LIPSSs, the LIPSSs can be divided into two types $[7,8]$. While the LIPSSs with periods approximately equal to the laser wavelength are called low spatial frequency LIPSSs (LSFLs), the LIPSSs with periods much smaller than the laser wavelength are referred to as high spatial frequency LIPSSs (HSFLs). Tomita et al. [9] fabricated a surface wave structure on a (100) silicon surface by a single femtosecond laser pulse. Their results show that the period of the surface wave structure is nearly equal to the wavelength of the irradiating 
laser pulse. Dufft et al. [10] fabricated LSFL with a period $(630-730 \mathrm{~nm})$ close to the wavelength and HSFL with a period of 200-280 nm by irradiating single-crystalline zinc oxide surfaces with multiple linearly polarized femtosecond pulses $(150-200 \mathrm{fs}, 800 \mathrm{~nm})$ in air. It is suggested that the period of LIPSSs can only be determined by the laser wavelength and the refractive index $[11,12]$.

However, laser pulse number, laser pulse energy, and laser scanning speed have been found to significantly influence the period of LIPSSs very recently [13-16]. Richter et al. [13] investigated the formation of self-organized nanostructures inside the bulk of fused silica induced by ultrafast laser pulses at high repetition rates. They found that the grating period continuously decreases with increasing number of pulses and finally saturates significantly below $\lambda / 2 n(\lambda$ is laser wavelength and $n$ is the refractive index of the irradiated material). Yang et al. [15] fabricated self-assembled, sub-wavelength periodic structures in fused silica by a tightly focused, linearly polarized femtosecond laser beam. Their results show that the period of the nanogratings decreases with increasing pulse energy and decreasing scanning speed in their study range.

In this paper, we report on the fabrication of periodic and uniform nanogratings on cemented carbide by femtosecond laser scanning. Specifically, three experiments are designed to study the influence of single pulse energy, scanning speed, and scanning spacing on the period and the uniformity of the formed nanogratings. The configurations of the nanogratings are characterized by scanning electron microscope (SEM) and scanning probe microscope (SPM).

\section{Experiments}

YS8 $(\mathrm{WC}+\mathrm{TiC}+\mathrm{Co})$ cemented carbide with the size of $16 \mathrm{~mm}$ x $16 \mathrm{~mm}$ x $5 \mathrm{~mm}$ were utilized for this study. The composition, physical, and mechanical properties of this material are shown in Table 1. The surface of this material was mechanically polished and washed in an ultrasonic cleaner with acetone for $30 \mathrm{~min}$. The surface roughness of the cemented carbide sample was less than $10 \mathrm{~nm}$. It was fixed on a three-dimensional motorized translation stage with a position accuracy of $100 \mathrm{~nm}$.

The cemented carbide sample was irradiated in air with linearly polarized, $800 \mathrm{~nm}, 120$ fs laser pulses from a Ti: sapphire regenerative amplified laser system (Coherent Inc.) operated at a repetition rate of $1 \mathrm{kHz}$. The polarization direction and power of the laser can be adjusted by a polarizer and an 
attenuator, respectively. The femtosecond laser pulses with single pulse energy of $1.75 \sim 2.75 \mu \mathrm{J}$ were focused through a microscope objective with a focal length of $23.5 \mathrm{~mm}$ and numerical aperture of 0.15 on the sample surface. The laser spot size was estimated to be $6.51 \mu \mathrm{m}$ in diameter and the laser fluence on the sample was estimated to be $2.28 \mathrm{~J} / \mathrm{cm}^{2}, 2.61 \mathrm{~J} / \mathrm{cm}^{2}, 2.94 \mathrm{~J} / \mathrm{cm}^{2}, 3.26 \mathrm{~J} / \mathrm{cm}^{2}$, and 3.59 $\mathrm{J} / \mathrm{cm}^{2}$, respectively. The scanning area was $0.4 \mathrm{~mm} \times 0.4 \mathrm{~mm}$. All tests were carried out with the following parameters: single pulse energy $E=1.75 \sim 2.75 \mu \mathrm{J}$, scanning speed $V=125 \sim 2000 \mu \mathrm{m} / \mathrm{s}$, scanning spacing $D=5 \sim 10 \mu \mathrm{m}$. The surface morphology of the sample after fs laser treatment was examined by scanning electron microscope (SEM). In addition, the amplitude of the fabricated nanogratings was also characterized by scanning probe microscope (SPM).

\section{Table 1}

Properties of the YS8 cemented carbide materials.

\begin{tabular}{ccccccc}
\hline $\begin{array}{c}\text { Composition } \\
(\text { wt. } \%)\end{array}$ & Flexural strength & Hardness & Density & Young's & Thermal expansion & Poisson's \\
\hline WC+TiC+Co & 1720 & 92.5 & 13.9 & 550 & coefficient $\left(10^{-6} / \mathrm{k}\right)$ & ratio \\
\hline
\end{tabular}

\section{Results and discussion}

The SEM pictures of the scanning area and the corresponding uniform nanogratings induced on YS8 cemented carbide by femtosecond laser are shown in Fig. 1(a) and (b), respectively. The EDX surface chemical composition analysis in the selected area point 1 is illustrated in Fig. 1(c). The single pulse energy, the scanning speed, and the scanning spacing are $2 \mu \mathrm{J}, 500 \mu \mathrm{m} / \mathrm{s}$, and $5 \mu \mathrm{m}$, respectively. $\mathrm{C}, \mathrm{W}, \mathrm{Co}$, and Ti elements are identified at point 1 of the uniform nanogratings (Fig. 1(c)), while the chemical composition of the substrate YS8 cemented carbide is the same. It is observed that uniform nanogratings on the surface of YS8 cemented carbide can be formed by femtosecond laser scanning without oxidation at certain processing parameters. The corresponding SPM plane-view and cross-section photos of the nanogratings in Fig. 1(b) are shown in Fig. 2(a) and (b), respectively. Figure 2(b) shows that the period of the uniform nanogratings is $\sim 650 \mathrm{~nm}$ and the groove depth of the nanogratings is $\sim 180 \mathrm{~nm}$. The direction of the uniform nanogratings is perpendicular to the laser polarization. 


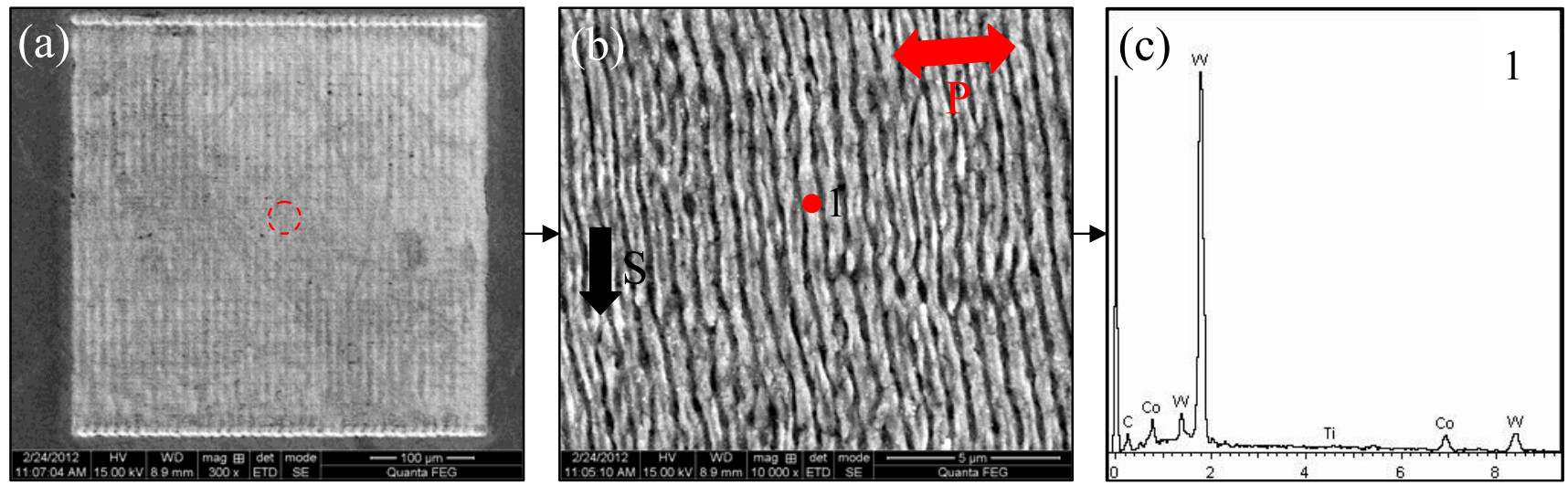

Fig. 1. (a) SEM photo of the scanning area at single pulse energy $E=2 \mu \mathrm{J}$, scanning speed $V=500 \mu \mathrm{m} / \mathrm{s}$, and scanning spacing $D=5 \mu \mathrm{m}$. (b) Magnified SEM photo of nanogratings corresponding to (a). (c) EDX composition analysis in the selected area point 1 of (b). $P$ denotes the orientation of the laser beam polarization. $S$ denotes the orientation of the laser scanning.
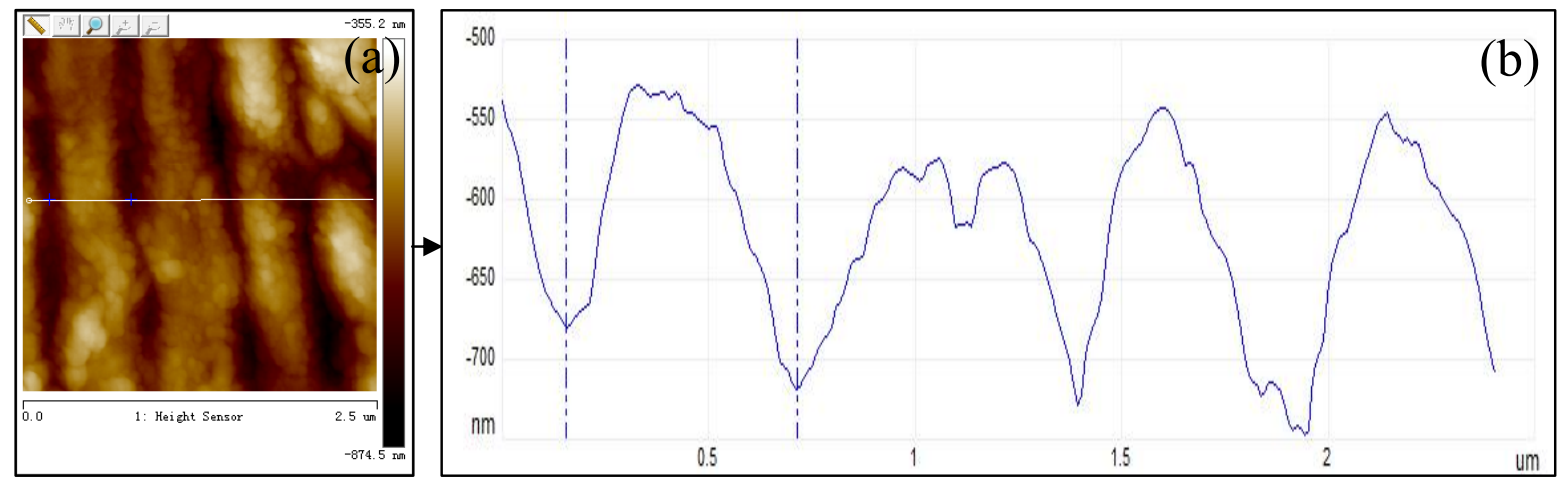

Fig. 2. (a) SPM plane-view photo of nanogratings at single pulse energy $E=2 \mu \mathrm{J}$, scanning speed $V=500 \mu \mathrm{m} / \mathrm{s}$, and scanning spacing $D=5 \mu \mathrm{m}$. (b) SPM cross-section photo of nanogratings corresponding to (a).

So far, several mechanisms have been proposed to explain the formation of nanogratings induced by femtosecond laser pulses, such as interference between the incident laser light and the surface scattered wave [17], self-organization [6], second harmonic generation (SHG) [8], excitation of surface plasmon polaritons [18], and Coulomb explosion [19] etc. However, it is suggested from our research that the surface plasmon polaritons (SPPs) excited by femtosecond laser irradiation can explain the formation of nanogratings in this paper [20,21]. The nanograting origin lies in the defect-initiated excitation of SPPs, which can interfere with the incident laser beam and then can lead to a modulated energy deposition into the YS8 cemented carbide [22].

\subsection{Single pulse energy}


It is known that nanogratings can be created on the surface of a material at certain single pulse energy [23-25]. Therefore, in order to investigate the dependence of the uniform nanogratings' configurations on single pulse energy, we designed the following experiments. We kept the scanning speed of $500 \mu \mathrm{m} / \mathrm{s}$ and the scanning spacing of $5 \mu \mathrm{m}$, while changing the single pulse energy from $2.75 \mu \mathrm{J}$ to $1.75 \mu \mathrm{J}$. Fig. 3 shows the SEM images of the nanogratings formed on the surface of YS 8 cemented carbide by femtosecond laser pulses with different single pulse energies and the EDX surface chemical composition analysis in the selected area. It can be seen that nanogratings with subwavelength periods can be obtained in all cases. However, a large area of the nanogratings is covered with some material with the single pulse energy of $2.75 \mu \mathrm{J}$, as shown in Fig. 3(a). The EDX surface chemical composition analysis on the covered area (point 1) is illustrated in Fig. 3(f). O, C, W, $\mathrm{Ti}$, and Co elements are identified in point 1 , while the nanogratings produced by femtosecond laser with single pulse energy of $2 \mu \mathrm{J}$ contain no $\mathrm{O}$ element as shown in Fig. 1(c). Apparently, high pulse energy resulted in the oxidation of the YS8 cemented carbide. In these cases, the surface of the YS8 cemented carbide was oxidized and formed nanogratings with ablated material adhered to the nanogratings (Fig. 3(a), (b), and (c)). The similar severe ablation was observed in previous research [24]. It also can be seen that the nanogratings appear to be short, irregular, and segmented for low single pulse energy as shown in Fig. 3(e). However, the sample with single pulse energy of $2 \mu \mathrm{J}$ shows the best uniform nanogratings among all the samples tested under different single pulse energies (Fig. 3(d)).

Fig. 4 shows the variations of nanograting period with single pulse energy (scanning speed $V=500$ $\mu \mathrm{m} / \mathrm{s}$, scanning spacing $D=5 \mu \mathrm{m})$. It can be seen from Fig. 4 that with the increase of single pulse energy from $1.75 \mu \mathrm{J}$ to $2.75 \mu \mathrm{J}$, the nanograting period decreases gradually from $\sim 681 \mathrm{~nm}$ to $\sim 431 \mathrm{~nm}$ and the gap between two adjacent peaks of the nanogratings gradually widens, as shown in Fig. 3 . The variations of nanograting period with single pulse energy are in agreement with previous observations [15]. However, the variability of the nanograting period is inconsistent with the simple nanoplasmonic model [26], which only allows for structures to be formed with a fixed period of $\lambda / 2 n$. Based on the theory of SPPs, nanograting period should somewhat smaller than the laser wavelength, 
since surface damage occurs at carrier concentrations close to the critical carrier density of SPP excitation [22]. And the variation of single pulse energy seems to change the carrier concentrations on the surface of YS8 cemented carbide, which leads to the change of nanograting period. The variation of the nanograting period in this study may be explained by the sensitivity of the nanograting period at excitations levels close to the critical carrier density/damage threshold. 


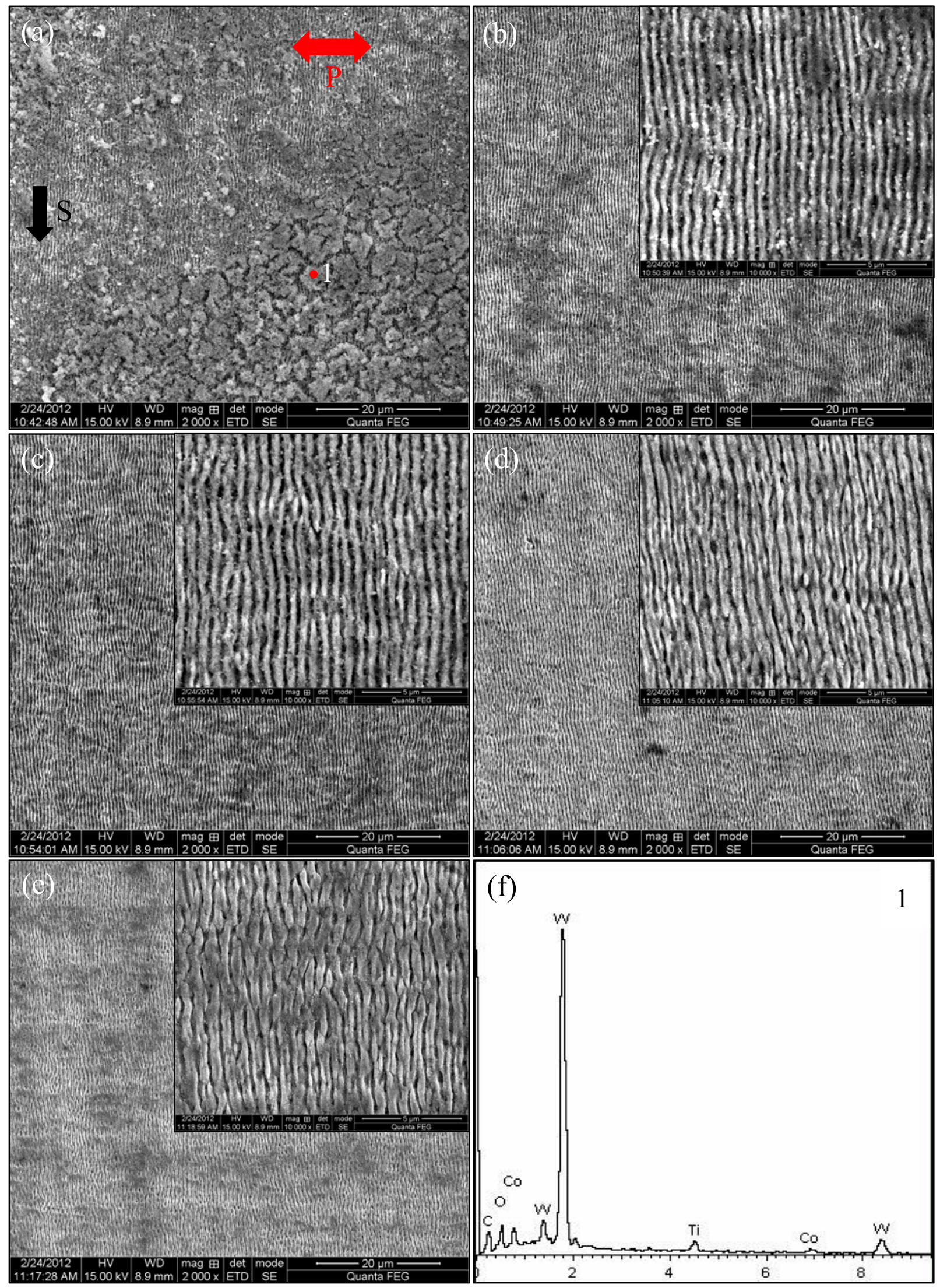

Fig. 3. SEM images of the nanogratings formed on the surface of YS8 cemented carbide by femtosecond laser pulses with different single pulse energies, (a) $2.75 \mu \mathrm{J}$, (b) $2.5 \mu \mathrm{J}$, (c) $2.25 \mu \mathrm{J}$, (d) $2 \mu \mathrm{J}$, (e) $1.75 \mu \mathrm{J}$, and (f) EDX 
composition analysis in the selected area point 1 of (a). $P$ denotes the orientation of the laser beam polarization. $S$ denotes the orientation of the laser scanning. (scanning speed $V=500 \mu \mathrm{m} / \mathrm{s}$, scanning spacing $D=5 \mu \mathrm{m}$ )

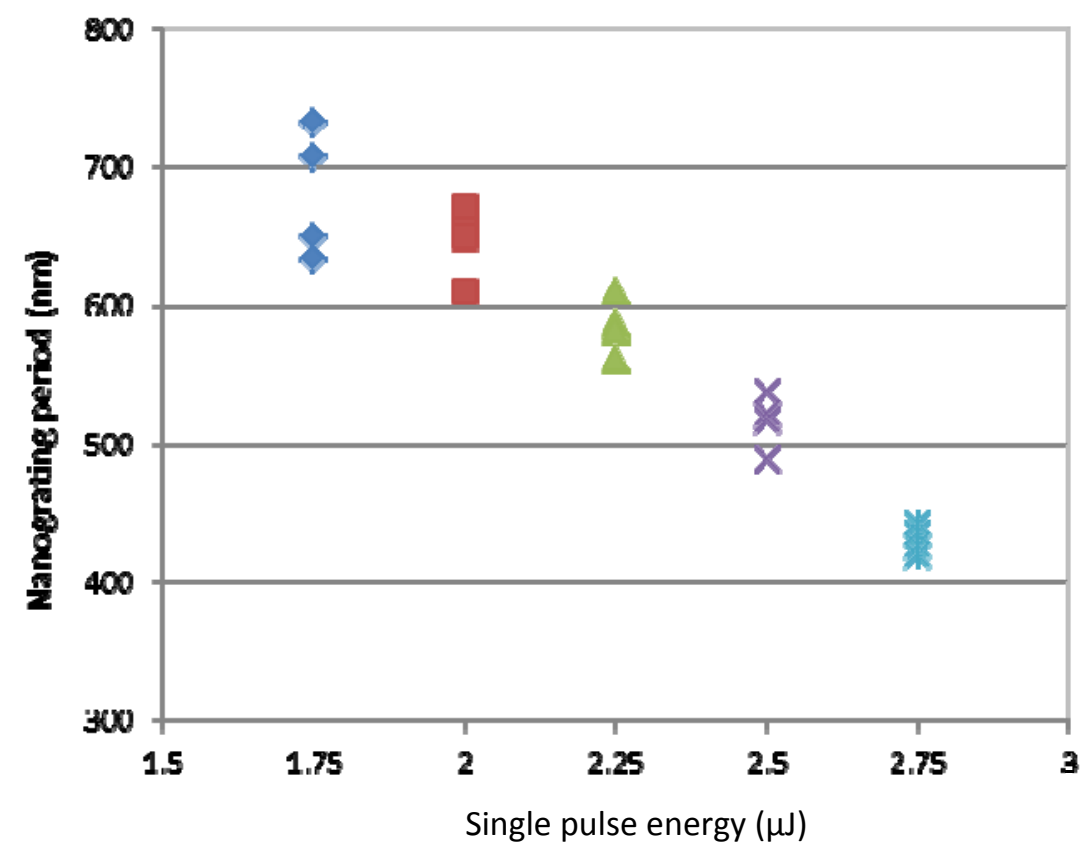

Fig. 4. Variations of nanograting period with single pulse energy. (scanning speed $V=500 \mu \mathrm{m} / \mathrm{s}$, scanning spacing $D=5 \mu \mathrm{m})$

\subsection{Scanning speed}

From the viewpoint of practical applications, it is essential to produce deep and uniform nanogratings that can be extended to a large area. Therefore, the scanning of focused laser beam is necessary. In this case, the single pulse energy, scanning speed, and scanning spacing will affect the quality of the formed nanogratings. So, in order to investigate the dependence of the uniform nanogratings' configurations on the scanning speed of the laser beam, we designed the following experiments. Based on the experimental results in Section 3.1, we kept the single pulse energy of $2 \mu \mathrm{J}$ and the scanning spacing of $5 \mu \mathrm{m}$, while changing the scanning speed from $2000 \mu \mathrm{m} / \mathrm{s}$ to $125 \mu \mathrm{m} / \mathrm{s}$.

Fig. 5 shows the SEM images of the nanogratings formed on the surface of YS8 cemented carbide by femtosecond laser pulses with different scanning speeds and the EDX surface chemical composition analysis in the selected area. It can be seen that nanogratings with subwavelength periods can be obtained in all cases. The sample with the scanning speed of $1000 \mu \mathrm{m} / \mathrm{s}$ shows long, straight, uniform, and regular nanogratings which are of the best quality among all the samples tested 
under different scanning speeds (Fig. 5(b)). However, the surface of YS8 cemented carbide can not form continuous nanogratings as a result of high scanning speeds such as $2000 \mu \mathrm{m} / \mathrm{s}$ (Fig. 5(a)). Meanwhile, certain areas of the nanogratings are covered with some dark material with the scanning speed of $125 \mu \mathrm{m} / \mathrm{s}$, as shown in Fig. 5(e). The EDX surface chemical composition analysis on the dark material (point 1) is illustrated in Fig. 5(f). O, C, W, Ti, and Co elements are identified in point 1 of the dark material, while the nanogratings produced with the scanning speed of $500 \mu \mathrm{m} / \mathrm{s}$ contain no O element as shown in Fig. 1(c). Apparently, at low scanning speeds too many pulses irradiated the same place and caused the oxidation of the material. In these cases, the surface of the YS8 cemented carbide was oxidized and formed nanogratings with ablated materials adhered to the surface of nanogratings (Fig. 5(d) and (e)). It also can be seen that the nanogratings appear to be thin, short, irregular, and segmented for low scanning speeds as shown in Fig. 5(d) and (e). Similar results were also found in previous research [27].

Fig. 6 shows the variations of nanograting period with scanning speed (single pulse energy $E=2$ $\mu \mathrm{J}$, scanning spacing $D=5 \mu \mathrm{m})$. It can be seen from Fig. 6 that with the increase of scanning speed from $125 \mu \mathrm{m} / \mathrm{s}$ to $2000 \mu \mathrm{m} / \mathrm{s}$, the nanograting period seems to increase gradually from $\sim 490 \mathrm{~nm}$ to $\sim 704 \mathrm{~nm}$ and the gap between two adjacent peaks of the nanogratings gradually narrows, as shown in Fig. 5. The approximately logarithmic dependence of the nanograting period on the scanning speed is in agreement with previous observations. Ramirez et al. [16] investigated the formation process of nanogratings in fused silica and the influence of fabrication parameters. Their results show that as the writing velocity is increased, the density of the nanogratings decreases as the mean spacing continuously grows. The variation of scanning speed seems to change the carrier concentrations on the surface of YS8 cemented carbide which leads to the change of nanograting period. The variation of the nanograting period may be explained by the sensitivity of the nanograting period at excitations levels close to the critical carrier density/damage threshold. 

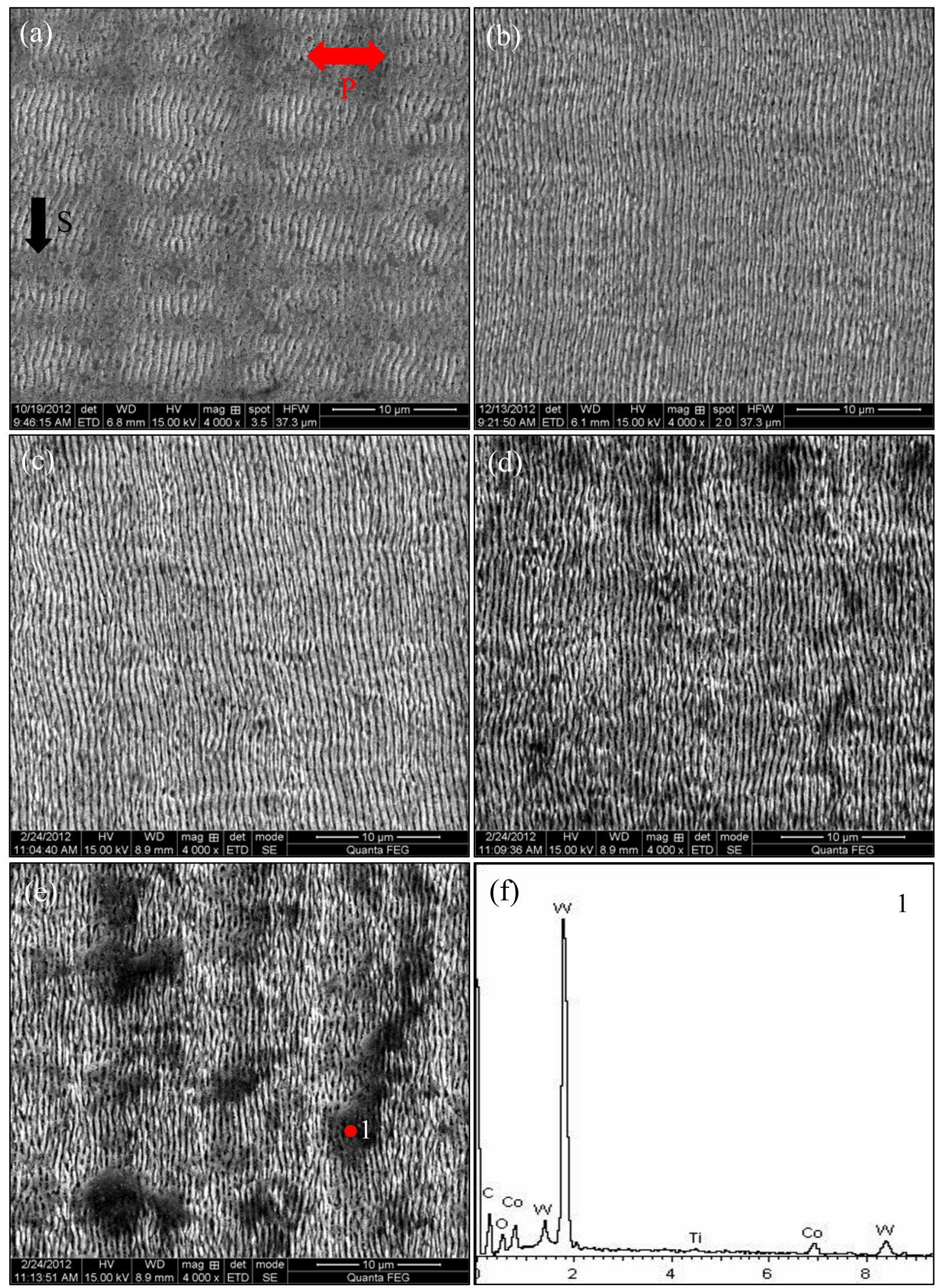

Fig. 5. SEM images of the nanogratings formed on the surface of YS8 cemented carbide by femtosecond laser pulses with different scanning speeds, (a) $2000 \mu \mathrm{m} / \mathrm{s}$, (b) $1000 \mu \mathrm{m} / \mathrm{s}$, (c) $500 \mu \mathrm{m} / \mathrm{s}$, (d) $250 \mu \mathrm{m} / \mathrm{s}$, (e) $125 \mu \mathrm{m} / \mathrm{s}$, and 
(f) EDX composition analysis in the selected area point 1 of (e). $P$ denotes the orientation of the laser beam polarization. $S$ denotes the orientation of the laser scanning. (single pulse energy $E=2 \mu \mathrm{J}$, scanning spacing $D=5$ $\mu \mathrm{m})$

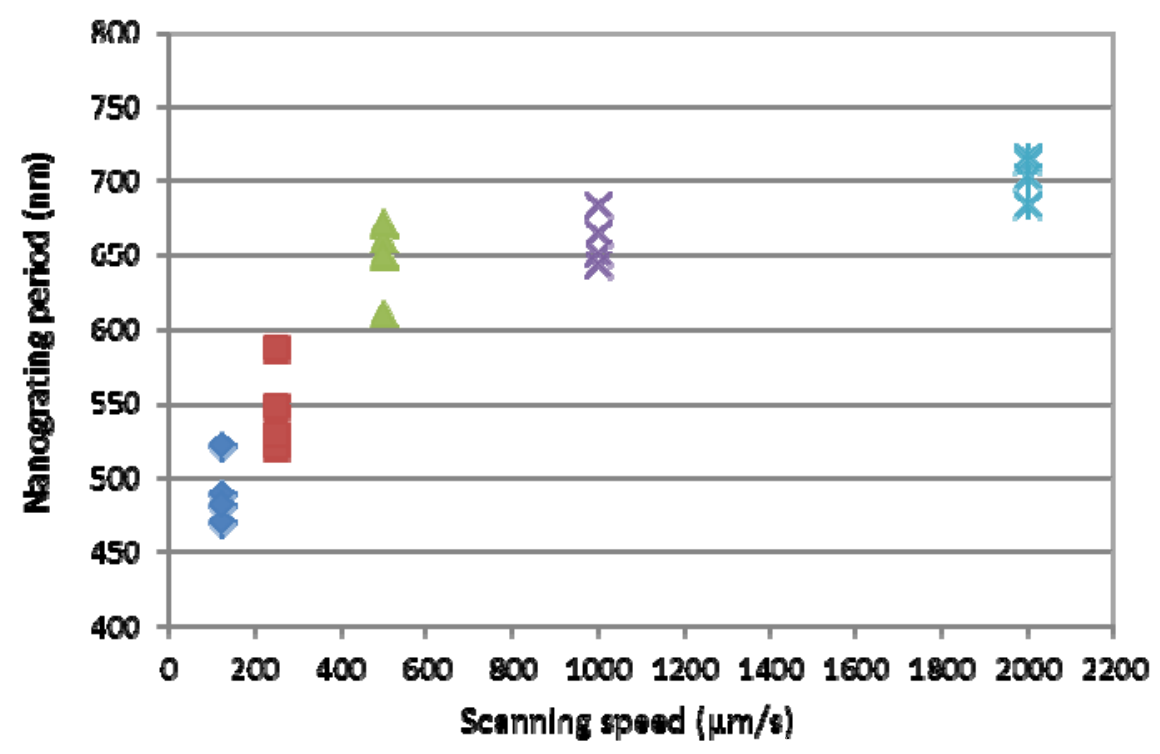

Fig. 6. Variations of nanograting period with scanning speed. (single pulse energy $E=2 \mu \mathrm{J}$, scanning spacing $D=5$ $\mu \mathrm{m})$

\subsection{Scanning spacing}

Another factor that determines the quality of the formed nanogratings is scanning spacing of the laser beam. Therefore, in order to investigate the dependence of the uniform nanogratings' configurations on the scanning spacing of the laser beam, we designed the following experiments. Based on the above experimental results, we kept the single pulse energy of $2 \mu \mathrm{J}$ and the scanning speed of $1000 \mu \mathrm{m} / \mathrm{s}$, while changing the scanning spacing from $5 \mu \mathrm{m}$ to $10 \mu \mathrm{m}$.

Fig. 7 shows the SEM images of the nanogratings formed on the surface of YS8 cemented carbide by femtosecond laser pulses with different scanning spacings and the EDX surface chemical composition analysis in the selected area. It can be seen that nanogratings with subwavelength periods can be obtained in all cases. The sample with the scanning spacing of $5 \mu \mathrm{m}$ shows continuous and uniform nanogratings which are of the best quality among all the samples tested under different scanning spacings (Fig. 7(a)). However, there are gaps between two laser scanning beams that can not 
form continuous nanogratings on the surface of YS8 cemented carbide, as shown in Fig. 7(b) and (c). The EDX surface chemical composition analysis on the gap (point 1) is illustrated in Fig. 7(d). C, W, Ti, and Co elements are identified on point 1 of the gap, while the chemical composition of the substrate YS8 cemented carbide is the same. Apparently, it can be said that if the scanning spacing is larger than the width of nanogratings formed by a single scan, it will cause the discontinuity of nanogratings formed on the surface. Conversely, it can be inferred that if the scanning spacing is smaller than the width of nanogratings formed by a single scan, it will cause the overlapping of nanogratings formed on the surface. It also can be found that the nanograting width formed by a single scan is about $5 \mu \mathrm{m}$ under the test conditions.

Table 2 shows the period of the nanogratings formed on the surface of YS8 cemented carbide by femtosecond laser pulses with different scanning spacings (single pulse energy $E=2 \mu \mathrm{J}$, scanning speed $V=1000 \mu \mathrm{m} / \mathrm{s})$. It can be seen that the nanograting period almost maintains a constant value of $\sim 660 \mathrm{~nm}$ under different scanning spacings. The gap between two adjacent peaks of the nanogratings also seems to be unchanged as shown in Fig. 7. It may be explained that the variation of scanning spacing seems not to change the carrier concentrations on the surface of YS8 cemented carbide which leads to the nanograting period unchanged. 

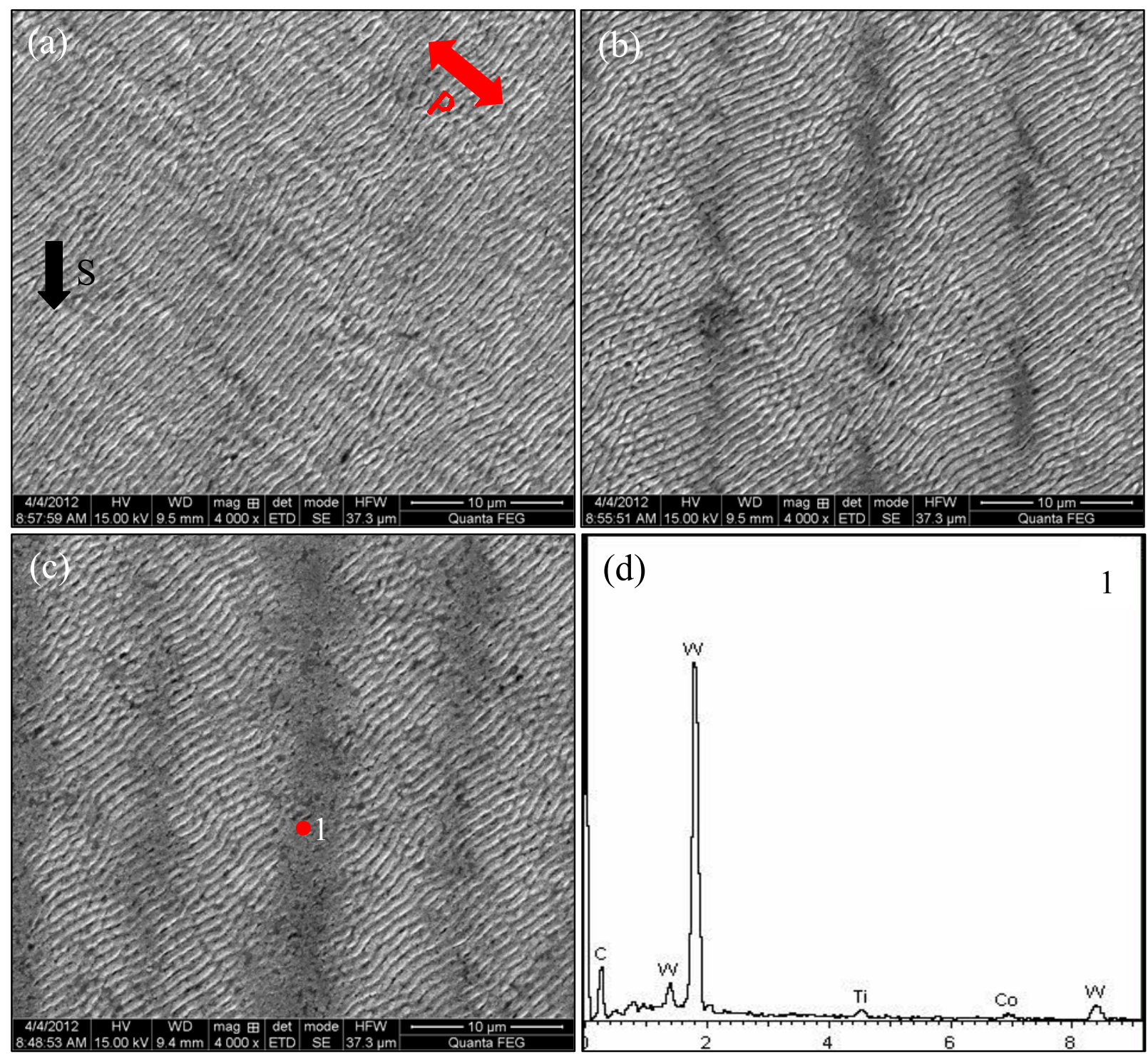

Fig. 7. SEM images of the nanogratings formed on the surface of YS8 cemented carbide by femtosecond laser

pulses with different scanning spacings, (a) $5 \mu \mathrm{m}$, (b) $8 \mu \mathrm{m}$, (c) $10 \mu \mathrm{m}$, and (d) EDX composition analysis in the selected area point 1 of (c). $P$ denotes the orientation of the laser beam polarization. $S$ denotes the orientation of the laser scanning. (single pulse energy $E=2 \mu \mathrm{J}$, scanning speed $V=1000 \mu \mathrm{m} / \mathrm{s}$ )

\section{Table 2}

Period of nanogratings formed on the surface of YS8 cemented carbide by femtosecond laser pulses with different scanning spacings. (single pulse energy $E=2 \mu \mathrm{J}$, scanning speed $V=1000 \mu \mathrm{m} / \mathrm{s}$ )

\begin{tabular}{cccc}
\hline Scanning spacing $(\mu \mathrm{m})$ & 5 & 8 & 10 \\
\hline Nanograting period $(\mathrm{nm})$ & 661 & 658 & 665 \\
\hline
\end{tabular}




\section{Conclusions}

In summary, we have successfully fabricated periodic and uniform nanogratings on cemented carbide by femtosecond laser scanning. Specifically, we designed three experiments to study the influence of single pulse energy, scanning speed, and scanning spacing on the period and the uniformity of the formed nanogratings. The main conclusions obtained can be summarized as follows.

(1) The sample with single pulse energy of $2 \mu \mathrm{J}$, scanning speed of $1000 \mu \mathrm{m} / \mathrm{s}$, and scanning spacing of $5 \mu \mathrm{m}$ shows the best nanograting quality among all the tested samples at different processing parameters.

(2) The uniformity of the nanogratings is largely determined by single pulse energy, scanning speed, and scanning spacing.

(3) Single pulse energy and scanning speed significantly affect the period of the nanogratings, whereas the period of the nanogratings maintains a fixed value under different scanning spacings. The period of the nanogratings increases gradually with the decrease of the single pulse energy and the increase of the scanning speed.

\section{Acknowledgments}

This work is supported by "National Natural Science Foundation of China (51075237)", "Taishan

Scholar Program of Shandong Province”, "Outstanding Young Scholar Science Foundation of Shandong (JQ200917)”, "Natural Science Foundation of Shandong (ZR2010EZ002)”, "Independent Innovation Foundation of Shandong University (2011JC001)" and "Specialized Research Fund for Doctoral Program of Higher Education (20110131130002)”.

\section{References}

[1] Q.Z. Zhao, S. Malzer, L.J. Wang, Formation of subwavelength periodic structures on tungsten induced by ultrashort laser pulses, Optics Letters 32 (2007) 1932-1934.

[2] S. Sakabe, M. Hashida, S. Tokita, S. Namba, K. Okamuro, Mechanism for self-formation of periodic grating structures on a metal surface by a femtosecond laser pulse, Physical Review B 79 (2009) 033409. 
[3] N. Yasumaru, K. Miyazaki, J. Kiuchi, Fluence dependence of femtosecond-laser-induced nanostructure formed on TiN and CrN, Applied Physics A 81 (2005) 933-937.

[4] X.D. Guo, R.X. Li, Y. Hang, Z.Z. Xu, B.K. Yu, H.L. Ma, B. Lu, X.W. Sun, Femtosecond laser-induced periodic surface structure on $\mathrm{ZnO}$, Materials Letters 62 (2008) 1769-1771.

[5] A.M. Ozkan, A.P. Malshe, T.A. Railkar, W.D. Brown, M.D. Shirk, P.A. Molian, Femtosecond laser-induced periodic structure writing on diamond crystals and microclusters, Applied Physics Letters 75 (1999) 3716-3718.

[6] F. Costache, M. Henyk, J. Reif, Modification of dielectric surfaces with ultra-short laser pulses, Applied Surface Science 186 (2002) 352-357.

[7] A.Y. Vorobyev, C. Guo, Femtosecond laser-induced periodic surface structure formation on tungsten, Journal of Applied Physics 104 (2008) 063523.

[8] A. Borowiec, H.K. Haugen, Subwavelength ripple formation on the surfaces of compound semiconductors irradiated with femtosecond laser pulses, Applied Physics Letters 82 (2003) 4462-4464.

[9] T. Tomita, Y. Fukumori, K. Kinoshita, S. Matsuo, S. Hashimoto, Observation of laser-induced surface waves on flat silicon surface, Applied Physics Letters 92 (2008) 013104.

[10] D. Dufft, A. Rosenfeld, S.K. Das, R. Grunwald, J. Bonse, Femtosecond laser-induced periodic surface structrues revisited: a comparative study on ZnO, Journal of Applied Physics 105 (2009) 034908.

[11] T.Q. Jia, H.X. Chen, M. Huang, F.L. Zhao, J.R. Qiu, R.X. Li, Z.Z. Xu, X.K. He, J. Zhang, H. Kuroda, Formation of nanogratings on the surface of a ZnSe crystal irradiated by femtosecond laser pulses, Physical Review B 72 (2005) 125429.

[12] T.Q. Jia, F.L. Zhao, M. Huang, H.X. Chen, J.R. Qiu, R.X. Li, Z.Z. Xu, H. Kuroda, Alignment of nanoparticles formed on the surface of $6 \mathrm{H}-\mathrm{SiC}$ crystals irradiated by two collinear femtosecond laser beams, Applied Physics Letters 88 (2006) 111117.

[13] S. Richter, M. Heinrich, S. Döring, A. Tünnermann, S. Nolte, Formation of femtosecond laser-induced nanogratings at high repetition rates, Applied Physics A 104 (2011) 503-507.

[14] E.M. Hsu, T.H.R. Crawford, H.F. Tiedje, H.K. Haugen, Periodic surface structures on gallium phosphide after irradiation with $150 \mathrm{fs}-7 \mathrm{~ns}$ laser pulses at $800 \mathrm{~nm}$, Applied Physics Letters 91 (2007) 111102. 
[15] W.J. Yang, E. Bricchi, P.G. Kazansky, J. Bovatsek, A.Y. Arai, Self-assembled periodic sub-wavelength structures by femtosecond laser direct writing, Optics Express 14 (2006) 10117-10124.

[16] L.P.R. Ramirez, M. Heinrich, S. Richter, F. Dreisow, R. Keil, A.V. Korovin, U. Peschel, S. Nolte, A. Tünnermann, Tuning the structural properties of femtosecond-laser-induced nanogratings, Applied Physics A $100(2010) 1-6$.

[17] J.E. Sipe, J.F. Young, J.S. Preston, H.M.V. Driel, Laser-induced periodic surface structure. I. Theory, Physical Review B 27 (1983) 1141-1154.

[18] G. Miyaji, K. Miyazaki, Origin of periodicity in nanostructuring on thin film surfaces ablated with femtosecond laser pulses, Optics Express 16 (2008) 16265-16271.

[19] Y. Dong, P. Molian, Coulomb explosion-induced formation of highly oriented nanoparticles on thin films of 3C-SiC by the femtosecond pulsed laser, Applied Physics Letters 84 (2004) 10-12.

[20] J. Wang, C. Guo, Formation of extraordinarily uniform periodic structures on metals induced by femtosecond laser pulses, Journal of Applied Physics 100 (2006) 023511.

[21] M. Huang, F. Zhao, Y. Cheng, N. Xu, Z.Z. Xu, Origin of laser-induced near-subwavelength ripples: between surface plasmons and incident laser, ACS Nano 3 (2009) 4062-4070.

[22] J. Bonse, A. Rosenfeld, J. Krüger, On the role of surface plasmon polaritons in the formation of laser-induced periodic surface structures upon irradiation of silicon by femtosecond-laser pulses, Journal of Applied Physics 106 (2009) 104910.

[23] L.T. Qi, K. Nishii, M. Yasui, H. Aoki, Y. Namba, Femtosecond laser ablation of sapphire on different crystallographic facet planes by single and multiple laser pulses irradiation, Optics and Lasers in Engineering 48 (2010) 1000-1007.

[24] Y.H. Han, S.L. Qu, Uniform self-organized grating fabricated by single femtosecond laser on dense flint (ZF6) glass, Applied Physics A 98 (2010) 167-170.

[25] Z.Y. Guo, S.L. Qu, L.L. Ran, S.T. Liu, Modulation grating achieved by two interfered femtosecond laser pulses on the surface of the silica glass, Applied Surface Science 253 (2007) 8581-8583.

[26] R. Taylor, C. Hnatovsky, E. Simova, Applications of femtosecond laser induced self-organized planar nanocracks inside fused silica glass, Laser \& Photonics Reviews 2 (2008) 26-46. 
[27] J.W. Yao, C.Y. Zhang, H.Y. Liu, Q.F. Dai, L.J. Wu, S. Lan, A.V. Gopal, V.A. Trofimov, T.M. Lysak, Selective appearance of several laser-induced periodic surface structure patterns on a metal surface using structural colors produced by femtosecond laser pulses, Applied Surface Science 258 (2012) 7625-7632. 